\title{
A Peer Review Writing Workshop in the Advanced Lab
}

\author{
Melanie B. Lott* \\ *Department of Physics \& Astronomy, Denison University, 100 W. College St., Granville, OH 43023
}

\begin{abstract}
At Denison University students in the advanced lab, PHYS 312: Experimental Physics, write substantial manuscript-style laboratory reports on three major experiments carried out during the semester. Thoughtful peer review can be an effective way to enhance student learning and confidence in their scientific writing skills. The development of purposeful assignments and subsequent class discussions of scientific writing and the peer review process, the execution of a peer review workshop, and samples of student work are presented in this paper.
\end{abstract}

Keywords: scientific writing, peer review, advanced lab

PACS: 01.40.gb

\section{INTRODUCTION}

Along with the goals of giving students experience
in designing experiments, developing their experimental and troubleshooting abilities, and refining their data analysis skills, we strive to teach students in the advanced lab at Denison University to become effective communicators of scientific findings. PHYS 312: Experimental Physics meets two days a week for three hours and on a third day for one hour. The class sessions focus on data and error analysis techniques and statistics, with some time devoted to mini-experiments. In the lab, students work in groups of 2 or 3 on three major experiments over the course of the semester and then write individual manuscriptstyle laboratory reports after each experiment.

The following paper describes the first PHYS 312 Peer Review Workshop carried out in the spring of 2015 and the preparation students received prior to the workshop. To set the stage for a successful peer review process, it is necessary to coach students how to provide helpful and effective feedback. ${ }^{1}$ This was accomplished primarily by devoting two 50-minute classes to scientific writing and peer review preparation leading up to the workshop.

The goals of the peer review were to enhance student learning of and confidence in their scientific writing skills. Though no direct measures of these two outcomes were taken, preliminary evidence in student comments and improvement of their reports after receiving peer feedback indicates that the peer review workshop was successful.

\section{PRELIMINARY ASSIGNMENTS}

Students completed assignments and specific class time was set aside early on with the intention to ease the transition from writing in other disciplines to the scientific manuscript writing style encountered in PHYS 312. The general timeline of these assignments and class discussions are shown in Table 1.

Each student received individual feedback on a draft of the first report in a one-on-one, 20-30 min meeting with the instructor. Written feedback on the draft was also provided. Students were given one week to make revisions before the report was due.

The second report was chosen to be peer-reviewed. At this point in the semester, after having one experimental report under their belts, students were more familiar with the style and expectations of the paper. Students also completed a "Peer Review of Writing" Prep assignment, and one class was devoted to discussing the peer review process (for details see the Peer Review of Writing Prep Assignment subsection).

TABLE 1. Timeline of Selected Writing Assignments

\begin{tabular}{c|l}
\hline Week & \multicolumn{1}{c}{ Assignment } \\
\hline 2 & "Science of Scientific Writing" \\
4 & Report 1 Drafts/ 1-on-1 instructor meeting \\
5 & Report 1 Due \\
8 & "Peer Review of Writing" Prep \\
9 & Report 2 Drafts/ Peer Review Workshop \\
10 & Report 2 Due \\
\hline
\end{tabular}

\section{"Science of Scientific Writing" Assignment}

For their first writing assignment, students read "The Science of Scientific Writing" by Gopen and $\mathrm{Swan}^{2}$ and were given the following prompt:

As you read the article "The Science of Scientific Writing," make two separate lists: (1) The things you think are important in the article and (2) the things you find interesting about the article. These should be typed, printed, and brought to class. They will be collected and graded as $+/$. 
At the beginning of class on the day the assignment was due, the students were instructed to hold onto their lists, and work in groups of 2-3 for $\sim 10$ minutes to come up with the group's "Top 4" most important things that they thought the authors sought to convey. Each group wrote their Top 4 list on a small whiteboard, and presented their list to the group, while the instructor compiled responses on the main whiteboard. The instructor then asked each of the thirteen students to share one thing they found interesting about the article.

The items in the students' Top 4 lists ranged from very specific and mechanical in nature to more general ideas about writing and included:

- Subject/ verb separation: keep the subject and verb of a sentence close.

- The stress position should be at the end of the sentence (new info/ emphasis).

- Limit the number of complete thoughts in a sentence.

- Limit the use of jargon and extreme number of acronyms.

- It is important to link the information that is presented and how it is related to the other information.

- Avoid logical gaps.

- Keep the audience in mind as you write.

- $\quad$ Reading = interpreting; Reader should come away interpreting what is important the same as the writer intended.

The Important/ Interesting exercise itself served to emphasize this last point.

One student mentioned that he thought the way the authors described the writing process as a way to "improve the quality of thought" was very interesting.

The instructor then shared her Top 4 list, some of which overlapped with the list compiled by the students. A few differences were noted. Many of the students focused on the more mechanistic points (e.g., subject/verb separation), which the instructor deemphasized. The other main difference was that the instructor thought an important take-away message of the article was that writing can often help the author's own thoughts evolve and refine. This was a very new concept for the students in the class even though many of us who have been writing as academics for some time have found that writing can advance and synthesize one's own thoughts.

The remaining class time was spent discussing the students' experience with reading scientific journal articles, and the format and style of the lab reports for PHYS 312, which are organized into the following sections: Abstract, Introduction, Theory, Experimental Methods, Data Analysis \& Discussion, Conclusions.

\section{Peer Review of Writing Prep Assignment}

One week prior to the Peer Review Workshop, students completed an assignment to prepare them for the peer review process. The goals of this assignment were to guide students to develop the framework for the peer review, discuss what types of feedback are helpful or not, and get them comfortable with giving and receiving critiques.

For this assignment, students read "Introducing Students to Peer Review of Writing" by Chisholm ${ }^{3}$ and completed an Important/ Interesting exercise with the same prompt that was used for the "Science of Scientific Writing" assignment. We began the class on the day the assignment was due with the same type of pair-and-share activity and creation of each group's Top 4 list and subsequent discussion. The following are the "important" points that students shared after reading the article:

- When asking someone to review your work, you should communicate your expectations for what type of feedback you hope to receive.

- $\quad$ Effective peer review $\neq$ Proofreading

- When reading someone's paper, the reviewer should consider the following:

- Is it appropriate for the audience?

- Are the motivation and hypothesis and/or goal of the experiment clearly stated?

- Does the author make connections to past information throughout the paper?

- Are there logical gaps? How much interpreting must be done?

- Is there an alternate way to analyze the data? Does the reviewer agree with author's choice of analysis?

- Is it clear how the error is quantified?

- Does the reviewer agree with the author's conclusion(s)?

- Asking the reviewer to describe/ summarize the paper can be an effective method to identify logical gaps and misinterpretation of your writing.

- The reviewer should provide both positive and negative feedback. It is important for the author to know what they are doing well and what can be improved.

- The feedback should be specific and constructive. Don't just say, "It looks good," or "I don't like this."

Complete drafts of Report 2 were due a few days after the Peer Review Prep assignment. As part of the draft, students were instructed to write a paragraph about what in particular they felt needed more work before the final paper was due, which was intended to 
help tailor some of the feedback they received from their peers. Each student's paper was reviewed by one peer. The instructor chose which students would peer review what papers with ability and class dynamics in mind. The reviewers had two days to read the paper, provide brief comments and notes within the paper, and type a 1-2 paragraph summary of their main comments (both positive and constructive criticism) for the author.

\section{PEER REVIEW WORKSHOP}

The peer review workshop began with a brief reminder to the students of the structure of the workshop. Students were to gather with their peer review group (five groups of 2 and one group of 3 ) and begin with either one of the papers, spending $\sim 20$ minutes per paper. They were to follow the guidelines set up based on the Chisholm article and peer review prep discussion in the following order:

1. Reviewer begins with a few positive aspects of the paper.

2. Reviewer describes/ summarizes the experiment and findings. (Is it consistent with author's intent?)

3. Reviewer and author discuss positive aspects and suggestions for improvement or rethinking (both writing and analysis methods).

4. Are there questions the reviewer has for the author or that the author has for the reviewer?

Naturally, as the students discuss the papers, there was some mixing of numbers 3 and 4, but peer reviewers were explicitly coached to spend the first few minutes simply describing the experiment and summarizing the results and main points before diving into the critique. This was a suggestion from the Chisholm article that we thought would be particularly helpful to identify any areas in which the reader misinterpreted the author's intended message, connecting back to Gopen and Swan's point that the author of a scientific paper should minimize readers' incorrect filling of logical gaps.

\section{Samples of Peer Feedback}

The following are samples of both positive and negative feedback from peer reviewers given in the 12 paragraph summaries they provided to the author of the paper they read.

Some comments concerned providing motivation:

"The motivation and goal are important

parts to the experiment, thus they need to be clarified at the beginning."
"Beginning with statistics of how dangerous gas and coal emissions can be is an excellent motivator for the reader. I found myself more interested in learning the history and theory of fuel cells later in the paper because of this."

Other comments identified places where descriptions were unclear or where a diagram would be helpful.

"The biggest improvement would be (besides actually calculating uncertainties) to reorganize and expand on the description of chaos and nonlinear motion."

"A circuit diagram would be very helpful in understanding how the load circuit is set up."

"I was thrown off by the mention that the first pendulum was made of two aluminum bars and would have liked to have seen a picture to better conceptualize it."

"How were you able to measure a phi of 0.77 degrees?"

Some students had trouble making an explicit connection between the variables in an equation they wrote in the paper with a conceptual explanation and/or the physical measurements they made in the experiment, which the following comment highlights:

"...give a better explanation of where the Lyapunov exponents come from. You give the equation, but show what factors in your experimental setup correspond to which variables in the equation."

Students provided less feedback to their peers on analysis methods, which partially appears to be a product of several students turning in drafts with very minimal data analysis. Below is an example of a positive comment concerning error analysis:

"The error analysis is inclusive as it covers a variety of uncertainties, both systematic and random, which demonstrates your particular attention to details and careful evaluation of experimental methods."

And finally, a positive comment about failed experiments:

"Even though the fuel cell gave you bad data, I appreciate that you discussed it and explained what it should have been and such. Sometimes bad data and failed experiments can still teach us something."

\section{Role of the Instructor in the Workshop}

Having led the students through all of the preparatory work and paired them in workshop groups that would hopefully set them up for a successful experience, the instructor's role in the workshop itself was minimal. The temptation to provide explicit content based or writing feedback was avoided even if the instructor had a strong opinion for or against 
comments provided by the reviewer. The peer review workshop presents an opportunity for students to not only receive feedback, but to assess the quality of that feedback, and decide for themselves what comments to take into account when writing their final report. If a student is given feedback that the instructor deems inappropriate, the instructor should discuss it with the student after the workshop. In addition, the students were given the option to set up a meeting with the instructor after the workshop to discuss the feedback they received and whether or not they agreed with all of it.

During the workshop the instructor circulated throughout the class to listen in on the discussions and help the students keep track of the time. There was one particular group that had a few disagreements that needed to be mitigated. The arguments dealt with stylistic and notational matters, so the students were instructed to agree to disagree and move on to more constructive conversation.

In general the students did extremely well and took the peer review workshop seriously and conducted it with enthusiasm. One sticking point for the entire class that the instructor had to address was to remind them to describe the experiment and summarize the paper before diving into the feedback. They were so eager to give and receive the critique that it was easy for them to forget this unusual, yet important, aspect of the process.

\section{After the Workshop}

At the end of the Workshop, the drafts with reviewer comments and summary paragraphs were returned to the authors. The students were given some time to reflect and consider which comments to use, what they still need to work on, or begin to make revisions immediately after receiving the feedback. Final versions of Report 2 were due one week following the workshop. Students handed in the drafts and reviewer comments with their papers so that the instructor could see how they used the feedback. About one-half of students met separately with the instructor after the workshop to receive feedback on their revised draft prior to handing in the final product.

\section{CONCLUSIONS}

The first PHYS 312 Peer Review Workshop went remarkably smoothly, and the students provided overall very thoughtful and useful feedback to their peers. After devoting only one class session specifically to preparing for the workshop, the peer reviews not only saved the instructor many hours of reading and providing feedback on individual drafts, the students may have benefited even more greatly from the peer review process than from receiving instructor feedback. Several students commented that they learned more about themselves as writers by reading and peer reviewing their classmates' papers. In addition, the second reports were on average the strongest reports of the three that were written. A study comparing students in the advanced lab who receive instructor feedback only and those that participate in a peer review workshop would be necessary to make a conclusive statement about the outcomes of increased confidence and improved scientific writing skills of students.

Several improvements could be made to the workshop such as putting the students in groups of three, with each person reading one other person's paper. This would present a built in mechanism to ensure that students remember to describe the experiment and its findings, with the reviewer summarizing the paper for the person in the group who had not read it. Additionally, providing a stronger incentive to hand in a complete draft for the peer review (e.g., an effect on the student's grade) may increase the feedback on analysis methods, yielding a more complete review and better overall outcome for the students.

\section{ACKNOWLEDGEMENTS}

To Dr. N. Daniel Gibson for sharing the Chisholm article that he has used to prepare students for written peer reviews of lab reports. To the Denison University Writing Committee and the Winter Writing Workshop for inspiring the author to formulate some of the ideas in developing the PHYS 312 Peer Review Workshop.

\section{REFERENCES}

1. J. C. Bean, Engaging Ideas, $2^{\text {nd }}$ ed (Jossey-Bass, San Francisco, 2011), pp. 295-302.

2. G. Gopen and J. Swan, Am. Sci.78, 550-558 (1990).

3. R. M. Chisholm, Writing Across the Curriculum 3, 4-19 (1991). 